\title{
EDITORIAL
}

\section{Los farmacéuticos comunitarios y la HTA}

\author{
Salvador Tous Trepati
}

Farmacéutico comunitario en Igualada (Barcelona). Grupo de HTA y riesgo vascular de SEFAC. Ipact. SCHTA. Grupo de trabajo en HTA de CAMFIC.

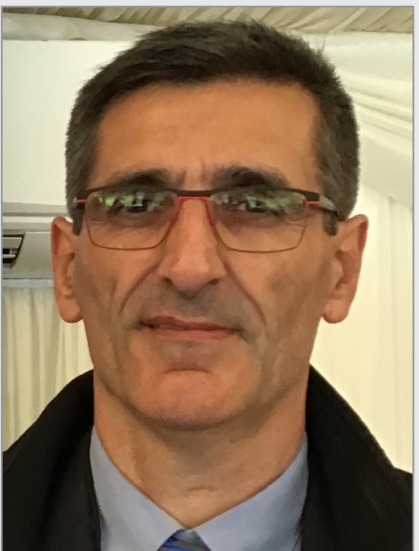

Salvador Tous Trepat

\section{PALABRAS CLAVE}

Hipertensión arterial, farmacéuticos comunitarios

\section{ABREVIATURAS}

AMPA: Automedida de la Presión Arterial

FC: farmacéuticos comunitarios HTA: hipertensión arterial MAPA: Monitorización Ambulatoria de la Presión Arterial

PA: presión arterial

\section{KEYWORDS}

High blood pressure, community pharmacists
La labor del farmacéutico comunitario en el paciente con hipertensión arterial (HTA) no suele tener repercusión en las publicaciones científicas por diferentes razones, entre ellas el poco hábito que tenemos los farmacéuticos comunitarios (FC) de registrar nuestra labor.

De todas formas, ya en la última Guía ESC de HTA $(1,2)$ aparecía remarcada por primera vez la función que los FC pueden hacer en este campo junto con la de los profesionales de enfermería. Y más teniendo en cuenta que en los últimos años la medida de la presión arterial ha ido descansando progresivamente en estos dos colectivos profesionales.

Aunque con diferencias, porque los FC podemos y debemos tener una labor insustituible en el cribado de los pacientes que desconocen que son hipertensos y el seguimiento de los pacientes ya diagnosticados, al menos los de baja o media complejidad.

La HTA es un problema que se presenta de forma progresiva y que puede acabar afectando a un porcentaje muy elevado de la población con implicaciones en su salud.

Hacemos y podemos hacer mucho, pero es muy importante que midamos la presión de forma incontestable, siguiendo los protocolos establecidos en cuanto a espacio, aparataje y procedimiento, siguiendo las últimas guías y al mismo tiempo registrando los datos. Plataformas como SEFAC e_XPERT facilitan enormemente el trabajo y permiten analizar los datos obtenidos, presentar informes al paciente y a los profesionales que los atienden, poniendo en valor nuestra labor y mejorando la eficiencia de nuestro trabajo.

Además, si como farmacéuticos comunitarios conseguimos registrar un volumen suficiente de datos, podríamos llegar a eliminar uno de los puntos grises de nuestra labor, que como se comenta en un artículo (3) publicado en este mismo número de FARMACÉUTICOS COMUNITARIOS, es establecer la equivalencia entre las medidas realizadas en la farmacia comunitaria y las realizadas en la consulta médica, que suponemos iguales, pero no existe evidencia científica publicada.

Por otro lado, no debemos olvidar nuestra labor promoviendo hábitos saludables, el cumplimiento de los tratamientos prescritos, los problemas asociados con su uso; existen multitud de problemas, percepciones y situaciones en las que podemos y debemos actuar, tal como se hace en el caso clínico también publicado en este número (4). Pacientes de alta complejidad en los que se han invertido muchos recursos públicos pueden poner en peligro su salud, por cuestiones que como farmacéuticos comunitarios podemos contribuir a resolver.

Volviendo a la calidad de las medidas, tal como se apunta $(1-3,5-7)$ existen herramientas a nuestro alcance como el AMPA (Automedida de la Presión Arterial), que requieren de la participación del paciente, pero que con la ayuda de plataformas como SEFAC e_XPERT podemos gestionar los datos obtenidos, pudiendo ofrecerlo incluso como un servicio renumerado y extraer conclusiones muy importantes sobre su grado de control como la presencia de HTA de bata blanca (circunstancia en absoluto banal y que puede ser tributaria de tratamiento o muchas veces antesala de HTA diagnosticada) o, aún peor, por el riesgo asociado de HTA enmascarada. 
0 yendo más allá, el increible artículo de Penin 0 et al (8) (también publicado en este número), por el número de pacientes reclutados, la complejidad de la técnica aplicada (aunque asumible, como demuestran los autores) y el periodo de seguimiento, muestra que no hay nada imposible si nos proponemos con determinación mejorar la salud de nuestros pacientes de forma proactiva. Los resultados de este artículo en concreto muestran el campo de trabajo en hábitos saludables como cesación tabáquica, que tenemos a nuestro alcance; inasumible por otros profesionales que no estén como nosotros a pie de calle día tras día. Además de las posibilidades que abren otros trabajos publicados por los mismos autores sobre el uso de MAPA (Monitorización Ambulatoria de la Presión Arterial) como herramienta en la farmacia comunitaria.

La barrera del conocimiento y experiencia de uso de estos procedimientos se ha tratado en programas como impacHta, del que esperamos próximamente una nueva edición que recoja lo recomendado en las últimas guías.

Para terminar una reflexión ligada con el momento histórico que nos toca vivir: estamos, parece, en la parte final de una pandemia que ha puesto, a nosotros incluidos, en jaque a todo el sistema sanitario. Muchos de nosotros suspendimos las medidas de PA en los momentos más duros de la pandemia y debimos tomar distancia de los pacientes. Ahora que todo poco a poco vuelve a la normalidad y entendiendo que el sistema sanitario público necesita tiempo para recomponer la atención habitual, es estratégico que los farmacéuticos comunitarios demos un paso al frente en una de las actividades asistenciales que más valoran nuestros pacientes, recuperando y promoviendo la toma de la presión arterial, siguiendo los protocolos e incorporando en la medida de nuestras posibilidades el servicio de AMPA o de MAPA a nuestra cartera de servicios. La salud de la población y la percepción que tienen de nuestra función lo agradecerán.

\section{REFERENCIAS BIBLIOGRÁFICAS}

1. Stergiou GS, Palatini P, Parati G, et al. 2021 European Society of Hypertension practice guidelines for office and out-of-office blood pressure measurement. Journal of Hypertension. 2021 Jul;39(7):1293-1302. doi:10.1097/HJH.0000000000002843

2. Mazon P (coordinador). Comentarios a la guia ESC/ESH 2018 sobre el diagnóstico y tratamiento de la hipertensión arterial. Rev Esp Cardiol. 2019;72(2):104-108. doi:10.1016/j.recesp.2018.11.022

3. García-Zaragoza E, Maria-Tablado MA. Comentario al nuevo Documento de Consenso de la Sociedad Europea de Hipertension 2021: "Guia Practicas sobre la Medida de Presión Arterial en Consulta y Ambulatoria. Farmacéuticos Comunitarios 2021; 13(4):21-27. doi:10.33620/FC.2173-9218.(2021/Nol13).004.04

4. Sanz Orejas MJ, Martinez Carretero E. Control de presión arterial en paciente doblemente trasplantado pluripatológico mediante servicios profesionales farmacéuticos asistenciales: protocolo $1 \mathrm{mpacH}-$ TA. Farmacéuticos Comunitarios 2021; 13(4):43-47. doi:10.33620/ FC.2173-9218.(2021/Nol13).004.06

5. Molinero Crespo A, Tous Trepat S, Pérez Fornos JA, Mera-Gallego I, Prats Mas R, Ruilope L. Detección de fenotipo hipertensión enmascarada en población adulta que acude a la farmacia comunitaria. Farmacéuticos Comunitarios 2020; 12 (Supl 2. Congreso SEFAC 2020):14 Disponible en: https://www.farmaceuticoscomunitarios.org/es/journal-article/deteccion-fenotipo-hipertension-enmascarada-poblacion-adulta-que-acude-farmacia

6. Prats R, Mera I, Tous S, Iracheta M, Molinero A, Perseguer Z, Andraca $L$, Rosinach J, Plaza J. Experiencia española en el proyecto 'MAY MEASUREMENT MONTH' 2017-2018-2019, desde la farmacia comunitaria. Farmacéuticos Comunitarios. 2020 Nov 05; 12 (Supl 2. Congreso SEFAC 2020): 35 Disponible en: https://www.farmaceuticoscomunitarios.org/es/journal-article/experiencia-espanola-proyecto-may-measurement-month-2017-2018-2019-desde-farmacia

7. Iracheta $M$, Catarineu $B$, Tous $S$. Automedida de la presión arterial (AMPA): herramienta de uso compartido entre diferentes profesionales sanitarios (caso clínico). Farmacéuticos Comunitarios. 11 (Supl $1^{\circ}$ Congreso Semergen SEFAC) Disponible en: https://www.farmaceuticoscomunitarios.org/es/journal-article/automedida-presion-arterial-ampa-herramienta-uso-compartido-entre-diferentes

8. Penín Álvarez O, Rojo Fernández JC, Penín Rodríguez A, Villasuso Cores B. Influencia de la dependencia del consumo de tabaco en el control de la presión arterial en personas con tratamiento farmacológico antihipertensivo. Farmacéuticos Comunitarios 2021; 13(4):5-11. doi:10.33620/FC.2173-9218.(2021/Nol13).004.02 